\title{
Unique MR Findings in a Case Series of Two Symptomatic Patients with Peliosis Hepatis
}

\author{
Drew Pierce1, Christopher S. Davis', Paul M. Bloomston², Zarine K. Shah' \\ ${ }^{1}$ Department of Radiology, Ohio State University Wexner Medical Center, Columbus, USA \\ ${ }^{2}$ Department of Surgery, Ohio State University Wexner Medical Center, Columbus, USA \\ Email: zarine.shah@osumc.edu
}

Received 28 December 2013; revised 28 January 2014; accepted 7 February 2014

Copyright (C) 2014 by authors and Scientific Research Publishing Inc.

This work is licensed under the Creative Commons Attribution International License (CC BY). http://creativecommons.org/licenses/by/4.0/

(c) (†) Open Access

\begin{abstract}
Purpose: The aim of this report is to describe the unusual MR imaging characteristics observed in two patients with biopsy-proven peliosis hepatis. Imaging findings using gadoxetate disodium (Eovist) as the contrast agent in a patient with peliosis hepatis are presented for the first time. Methods: This is a retrospective review of the MRI findings in two patients reviewed independently by two specialized abdominal imaging radiologists. The radiological findings were correlated with clinical history and histopathology. Results: Peliosis hepatis is a rare clinical and radiological entity that is often a diagnostic dilemma due to its non-specific clinical characteristics. Unusual imaging characteristics in this rare entity make diagnosis even more challenging. Conclusions: Improved understanding of the imaging characteristics of peliosis hepatis may prevent unnecessary and potentially dangerous biopsies in select patients with peliosis hepatis. This requires a high index of suspicion for practicing radiologists due to the rarity of this disease.
\end{abstract}

\section{Keywords}

Peliosis Hepatis; Magnetic Resonance Imaging; Hepatic lesions; Liver Disease; Gadoxetate Disodium

\section{Introduction}

Peliosis hepatis is an uncommon clinical entity characterized by irregular blood-filled cavities in the liver. Since the term was coined by Schoenlank in 1916, only case reports have been published, leaving the condition poorly understood. Suggested etiologies include infection, malignancy, immunosuppressive states, and drug toxicity. Liver failure and life-threatening intra-abdominal hemorrhage are potential complications [1], [2]. Diagnosis of peliosis hepatis is often delayed due to its rarity and vague clinical manifestations [3]. Here, we present the cases 
of two symptomatic patients with special attention paid to the atypical computed tomography (CT) and magnetic resonance imaging (MRI) findings. The MR imaging findings with the use of gadoxetate disodium, a hepatocyte-specific contrast agent, are described for the first time.

\section{Case History, Patient 1}

A 41-year-old woman with a history of hypertension and fibromyalgia presented to her primary care provider with vague epigastric pain and nausea, which began approximately one week prior. The patient's medications included atenolol, carisoprodol, and a birth control pill (levonorgestrel-ethinylestrad). An ultrasound performed three years prior revealed fatty infiltration of the liver and a single hemangioma. This study was not available for review. Initial liver function tests were within normal ranges.

Multiphasic CT done at an outside institute which revealed numerous hypodense lesions of various size on the unenhanced scan. There was intense arterial enhancement of all the lesions, with the larger lesions being hypodense centrally. The lesions become nearly isodense to the background liver on the venous and delayed phases.

A noncontrast and contrast-enhanced CT revealed numerous $5 \mathrm{~mm}-2 \mathrm{~cm}$ hypodense lesions scattered throughout the liver. Most of the lesions enhanced during the arterial phase but the largest of the lesions remained hypodense centrally, with filling on delayed images (Figure 1). This was thought to represent the patient's previously-known hemangiomas.

Abdominal MRI (Figure 2) revealed numerous 1 - $2 \mathrm{~cm}$ T2 hyperintense lesions scattered throughout the parenchyma of the liver. The lesions were not well visualized on precontrast T1 images. Administration of intravenous gadolinium revealed subtle rim-enhancement in some of the lesions on the arterial phase. Subsequent venous, equilibrium, and delayed-phase images revealed many more enhancing lesions than could be visualized during the arterial phase. The lesions are well visualized on diffusion-weight scans as multiple hyperintensities.

\section{Case History, Patient 2}

A 37-year-old woman presented with acute onset of epigastric and right upper quadrant pain, with associated nausea, vomiting and exacerbated gastroesophageal reflux. Her past medical history included hypertension, obesity, gastroesophageal reflux, and a fifteen-year history of smoking. There was no history of prior malignancy or hepatic disease. Her medications included cyclobenzaprine, hydrochlorothiazide, esomeprazole, ranitidine, bupropion, lisinopril, ibuprofen, zolpidem tartrate, sucralfate, and an oral contraceptive pill. Initial laboratory investigations were within normal limits, with the exception of a mildly elevated alkaline phosphatase.

Abdominal ultrasound performed at an outside hospital showed multiple hypoechoic lesions, one of which was $5.6 \mathrm{~cm}$ in greatest dimension. Follow-up CT (Figure 3) showed multiple enhancing lesions throughout the liver (Figure 3). These lesions were very subtle on noncontrast CT and were seen as focal areas of altered attenuation on narrow window settings.

MRI of the abdomen was performed (Figure 4) which revealed a background of hepatic steatosis (based on signal drop on the opposed phase scan in (Figure 4(e)) with multiple hetererogeneously hyperintense lesions on T2-W imaging that were isointense on in-phase T1-W scan with hyperintense signal in out-of phase imaging and fat-suppression. Scans were performed using hepatocyte specific contrast agent gadoxetate disodium with the addition of the 20 minute delayed scans. The lesions enhanced on the arterial phase with Eovist ${ }^{\circledR}$ (gadoxetate disodium) contrast with some internal heterogeneity in the larger lesions. There was some central decrease in enhancement on the venous and delayed phases. All the lesions except for 2 (one in segment 5 and the other in segment 3) were hypointense to the liver on the 20 minute delayed scan after gadoxetate-disodium contrast administration. Diffusion imaging revealed that the lesions were bright on DWI and were not dark on the ADC maps.

Laparoscopic biopsy was performed after a percutaneous biopsy failed to produce diagnostic tissue. Multiple dark lesions scattered about the surface of the liver were seen during the procedure and frozen sections of these lesions were obtained. Pathology revealed prominent focal sinusoidal dilatation consistent with peliosis hepatis, along with moderate steatosis and mild chronic portal inflammation (Figure 5). Follow-up imaging was performed at 3 months and 1 year interval showed no significant interval change in imaging morphology of any of the lesions, despite the complete resolution of in the patient's symptoms. 


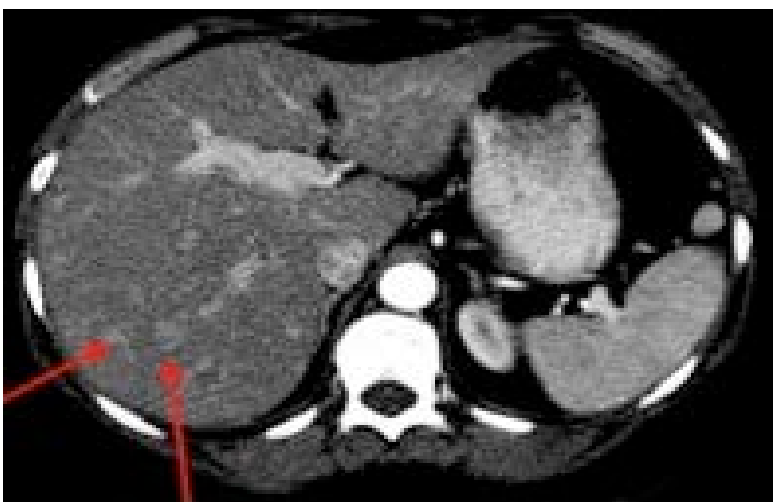

(a)

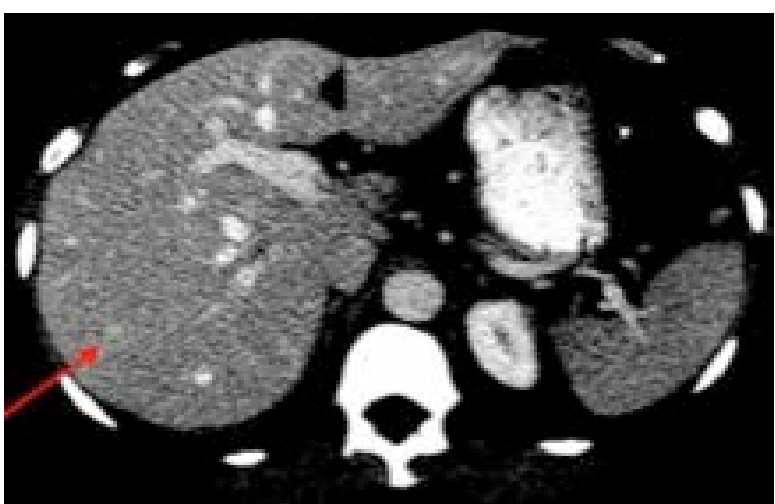

(b)

Figure 1. Contrast-enhanced CT, in the arterial (a) and portal-venous phase (b). Lesions of various sizes and morphology are seen throughout the liver. Most of the lesions enhance during the arterial phase (a) and become almost isodense on the portal venous phase (b).

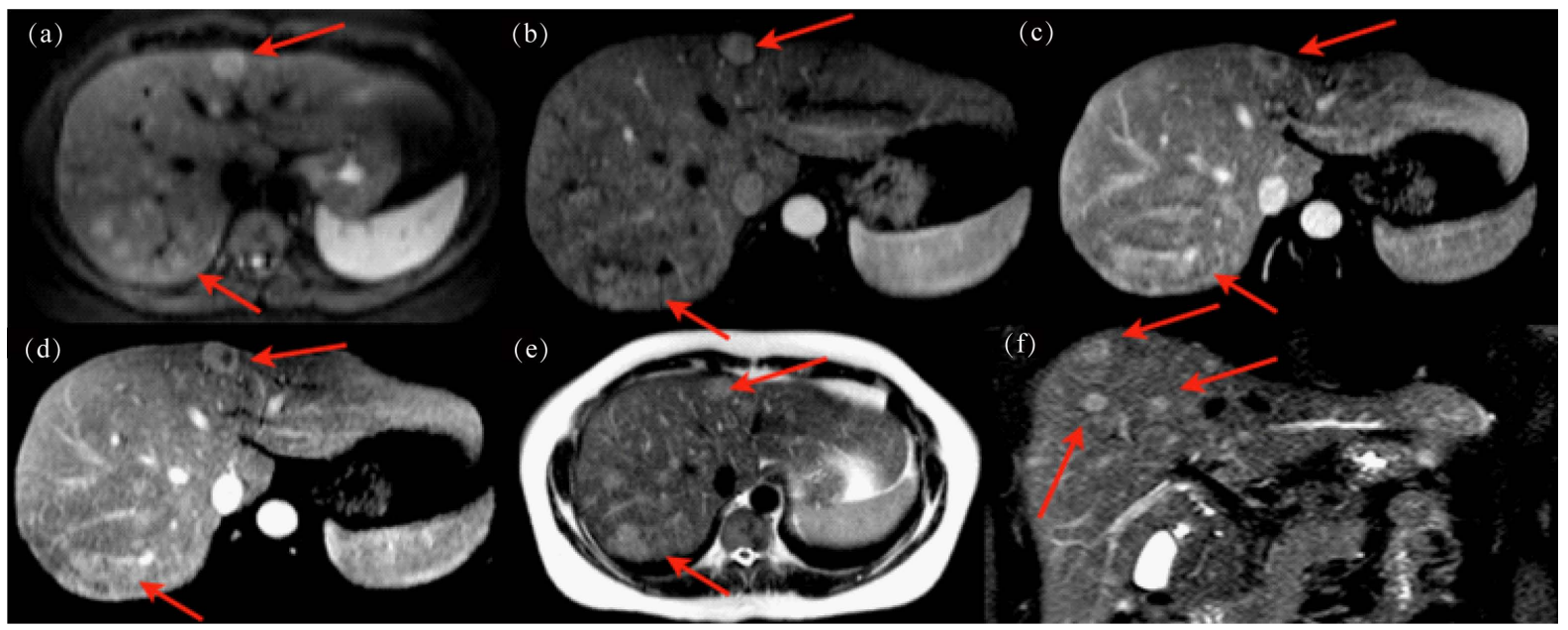

Figure 2. Multi-sequence MRI with and without gadolinium. (a) Diffusion weighted axial image shows lesions with restricted diffusion (b). Arterial enhancing phase images show early enhancement (c). Early and (d) late venous phase contrast enhanced axial images demonstrate avid enhancement. (e) T2 weighted axial and (f) coronal images demonstrate increased T2W signal within the lesions.

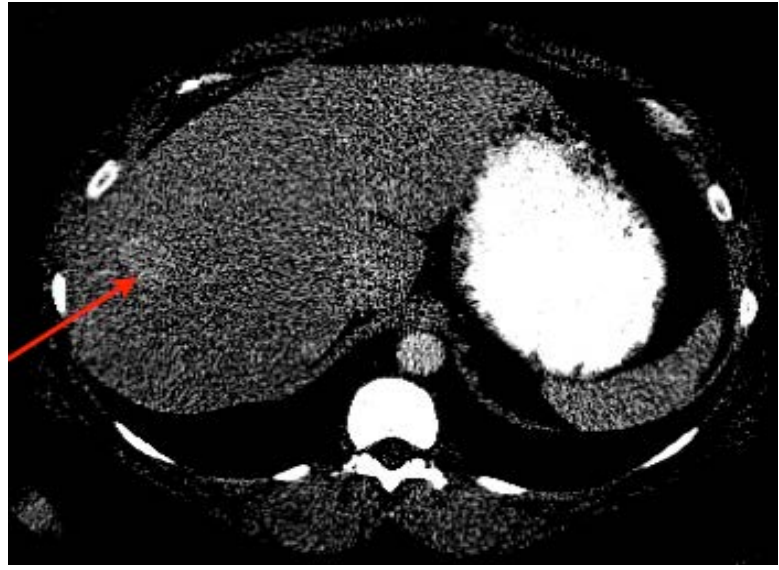

(a)

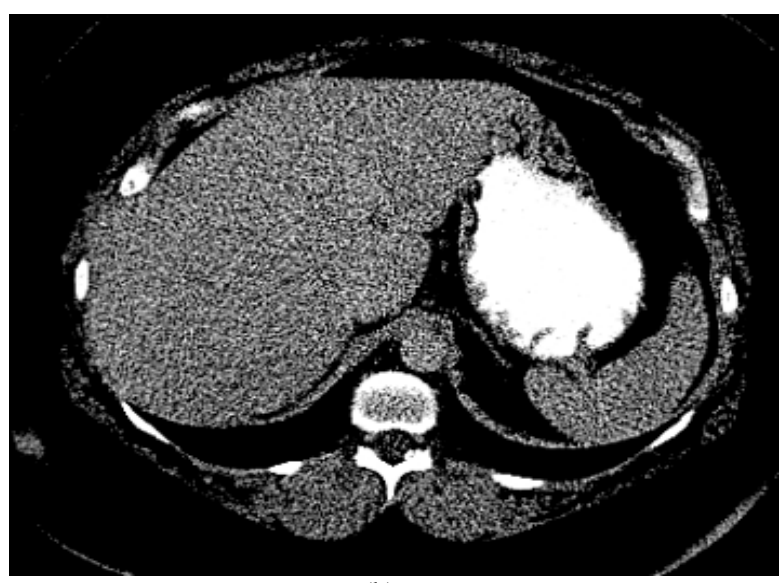

(b)

Figure 3. Venous (a) and delayed phase (b) contrast-enhanced CT revealing multiple lesions with faint contrast enhancement on the venous phase becoming isodense on the delayed phase. 


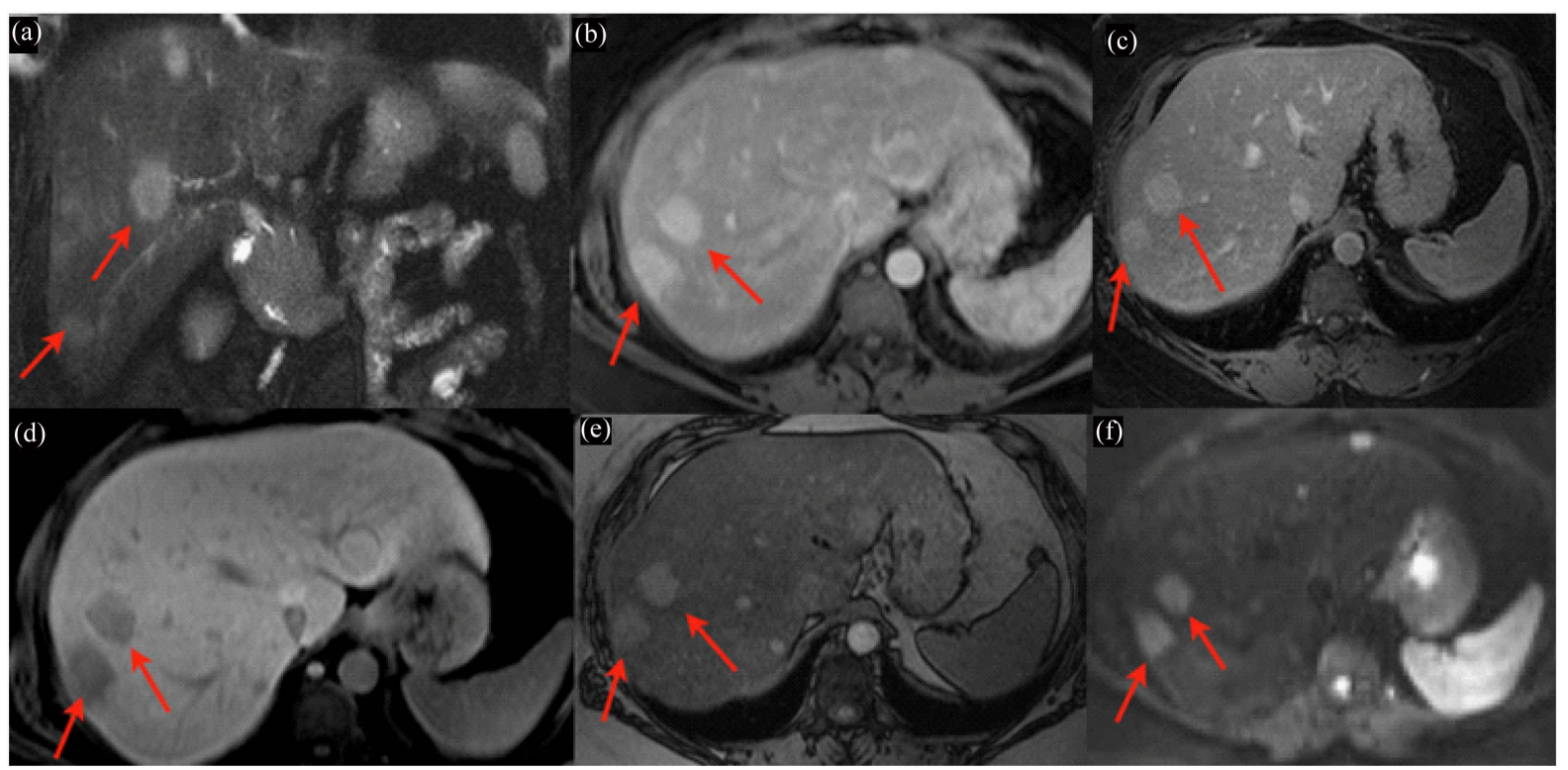

Figure 4. Multi-sequence MRI with and without Eovist ${ }^{\circledR}$ (gadoxetate disodium) contrast. (a) Coronal T2W MR image shows multiple hyperintense lesions. (b), (c) \& (d) T1-W Contrast enhanced images in the arterial, venous and 20 minute delayed phases respectively show multiple lesions with avid early enhancement that decreases below background on delay. (e) Opposed phase axial MR image shows increased signal within the lesions and decreased signal in background liver. (f) Diffusion Weighted axial image shows restricted diffusion in the lesions.

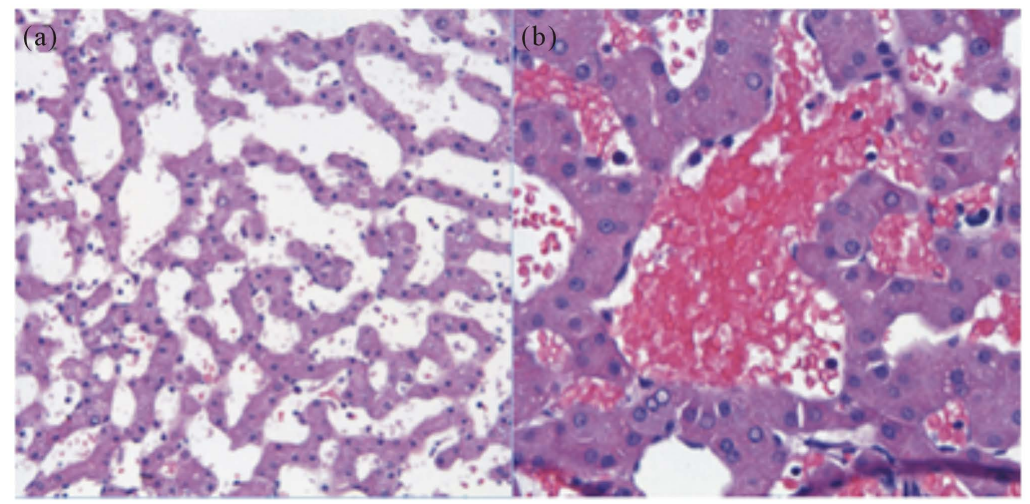

Figure 5. Pathology reveals sinusoidal dilatation ((a), HE $\times 20)$ and multiple blood-filled cystic spaces in the liver parenchyma ((b), HE $\times 40)$, characteristic of peliosis hepatis.

\section{Discussion}

Peliosis hepatis is an uncommon and potentially life-threatening clinical entity characterized by irregular bloodfilled cystic cavities in the liver. The word derives from the Greek pelios, which translates to "bluish-black." The term was first used by Schoenlank in 1916 in describing the liver of a 33-year-old woman who died from miliary tuberculosis. Peliosis has also been described in other organs belonging to the mononuclear phagocytic system (MPS), such as the spleen, bone marrow, and lymph nodes.

Two pathologic categories of peliosis hepatis have been described, the "phlebectatic" type, in which the cavities are lined by endothelium, and the "parenchymal" type, in which the cavities are lined by necrotic hepatocytes [1]. The pathogenesis of peliosis hepatis remains unclear, although hepatocellular toxicity, sinusoidal outflow obstruction, and endothelial toxicity have been suggested as potential contributing factors.

Proposed etiologies include malignancy (i.e. lymphoma and HCC), infection (i.e. HIV and Bartonella species), immunosuppressive states (i.e. solid organ transplants), and drug toxicity (particularly oral contraceptives 
and androgenous steroids). No specific cause is identified in 20\% - 50\% of patients [1]. Both patients in our report were taking oral contraceptives and experienced symptomatic improvement after their cessation, making this a possible etiologic agent. Both patients in our series were also taking muscle relaxants, which have been implicated in liver toxicity but not yet in the development of peliosis hepatis.

Complications described in this condition include liver failure, portal hypertension, expanding hematoma, and life-threatening intra-abdominal hemorrhage requiring surgical intervention [1], [2]. The natural history is typically regressive after withdrawl of the offending agents or treatment of the underlying causative infection [3]. Due to lack of specific clinical symptoms and because of its variable appearance and rarity, the diagnosis of peliosis is often significantly delayed [1].

It has been suggested in the literature that peliosis is typically asymptomatic unless complications arise. The patients presented in our report sought medical care for severe abdominal pain and nausea. Like the case described by Gouya et al., our patients did not show regression on follow-up imaging, however the patients became asymptomatic clinically.

Definitive diagnosis can be achieved by ultrasound-guided needle aspiration, although laparotomy may be safer and more effective option for many patients [1]. It has been suggested previously that biopsy not be performed on patients with risk factors and characteristic imaging findings due to the small but well-documented possibility of severe hemorrhage.

The imaging characteristics of peliosis are variable, as only case reports have been published to date. The imaging differential includes hepatic adenoma, hemangioma, arterial venous malformation, vascular metastasis, primary liver neoplasm, hepatic abscesses, and focal nodular hyperplasia. Peliotic lesions may be differentiated by their absence of mass effect, centrifugal enhancement pattern, and early and persistent filling on contrastenhanced imaging [4]. Hemangiomas typically have mass effect and a centripetal pattern of contrast enhancement [5].

On unenhanced CT imaging, peliotic lesions are typically hypoattenuating, although heterogenous and hyperattenuating lesions may be seen if acute blood is present [4]. Contrast-enhanced CT imaging is highly variable, and depends on the size of the peliotic lesions, the timing of intravenous contrast administration, and the presence of acute or sub-acute blood. The most typical pattern is early central arterial phase enhancement of lesions with centrifugal progression of contrast in the portal venous phase [3]. The lesions described in our patients did not follow this pattern.

On T1-weighted MR, lesions are typically hypointense, although isointense and hyperintense lesions have been described [6], [7]. Enhancement is nearly always seen following contrast administration, and follows a centrifugal pattern [3]. On T2-weighted imaging, lesions are nearly always hyperintense to the liver parenchyma and restricted diffusion may be seen [7]. MRI of peliotic lesions using Eovist ${ }^{\circledR}$ (gadoxetate disodium) as the contrast agent has not been described previously. In this case, we observed arterial enhancement with mild washout on the venous phase. On the 20-minute delayed phase, we observed predominately hypoattenuating lesions, suggesting paucity of functioning hepatocytes. A few a lesions demonstrated persistent high signal on the 20-minute phase, which could represent an element of cholestasis in these lesions.

\section{Conclusion}

Peliosis hepatis is a rare but potentially life-threatening clinical entity. Although it is a rare entity, peliosis hepatis should be kept in the differential diagnosis for all hepatic lesions of unclear etiology. Because the disease can be fatal and is potentially treatable, it is important to be familiar with its radiologic manifestations. In this case, we observed an enhancement pattern in which peliotic lesions enhanced markedly during the arterial and venous phases but appeared predominately hypoattenuating on delayed-phase imaging using a hepatocyte-specific contrast agent. This specific contrast agent use has not yet been described in this entity.

\section{References}

[1] Tsokos, M. and Erbersdobler, A. (2005) Pathology of Peliosis. Forensic Science International, 149, 25-33. http://dx.doi.org/10.1016/j.forsciint.2004.05.010

[2] Choi, S.K., Jin, J.S., Cho, S.G., Choi, S.J., Kim, C.S., Choe, Y.M. and Lee, K.Y. (2009) Spontaneous Liver Rupture in a Patient with Peliosis Hepatis: A Case Report. World Journal of Gastroenterology, 15, 5493-5497.

http://dx.doi.org/10.3748/wjg.15.5493 
[3] Iannaccone, R., Federle, M.P., Brancatelli, G., et al. (2005) Peliosis Hepatis: Spectrum of Imaging Findings. American Journal of Roentgenology, 187, W43-W52. http://dx.doi.org/10.2214/AJR.05.0167

[4] Kleinig, P., Davies, R.P., Maddern, G. and Kew, J. (2003) Peliosis Hepatis: Central "Fast Surge" Ultrasound Enhancement and Multislice CT Appearances. Clinical Radiology, 58, 995-998. http://dx.doi.org/10.1016/S0009-9260(03)00141-7

[5] Gouya, H., Vignaux, O., Legmann, P., et al. (2001) Peliosis Hepatis: Triphasic Helical CT and Dynamic MRI Findings. Abdominal Imaging, 26, 507-509. http://dx.doi.org/10.1007/s00261-001-0023-X

[6] Verswijvel, G., Janssens, F., Colla, P., et al. (2003) Peliosis Hepatis Presenting as a Multifocal Hepatic Pseudotumor: MR Findings in Two Cases. European Radiology, 13, L40-L44. http://dx.doi.org/10.1007/s00330-003-1852-5

[7] Battal, B., Kocaoglu, M., AvniAtau, A. and Bulakbasi, N. (2010) Multifocal Peliosis Hepatis: MR and DiffusionWeighted MR Imaging: Findings of an Atypical Case. Upsala Journal of Medical Sciences, 115, 153-156. http://dx.doi.org/10.3109/03009730903262118 